\title{
The Gaussian cannon
}

\author{
Åke Andersson ${ }^{*}$, Carl-Joar Karlsson, and Hampus Lane \\ Chalmers University of Technology, Göteborg, Sweden
}

Received: 2 August 2017 / Accepted: 4 August 2017

\begin{abstract}
This report studies different properties of a Gaussian cannon. The cannon's firing velocity is measured for a varying number of steel balls, varying input velocity and different geometries of the cannon. By measuring the attractive force from the magnet on the balls a mapping of the potential energy for the firing process is acquired. It is found that the firing of a Gaussian cannon can be modeled as successive collisions between the magnet and its neighboring balls, and between successive balls. To reach the highest possible ejection speed with a trigger ball that is released from rest, a chained cannon should be used. The optimal number of balls on each individual cannon depends on magnetization energies and coefficients of restitution.
\end{abstract}

Keywords: Gaussian cannon / cannon / magnet / dynamics / chain

\section{Introduction}

The Gaussian cannon is a popular device for teaching since it is easy to construct and affordable. It is constructed as seen in Figure 1. A line of steel balls are attached to a strong magnet. If an additional ball collides with the magnet the outermost ball can be ejected at high speed. In the presence of a magnetic field the colliding ball, or trigger ball, loses magnetic potential energy and gains kinetic energy. By putting more balls on the firing side of the magnet the potential energy lost by the trigger ball is made smaller than the potential energy gained by the ejected ball. Hence the ejected ball can reach higher speeds than the trigger ball. However, due to energy losses in collisions the ejection speed will not increase indefinitely. Thus the Gaussian cannon is a good example of a system which can be optimized.

Though the Gaussian cannon is easily constructed, a thorough analysis is far from trivial due to the sheer amount of parameters. This report focuses on determining the speed of the ejected ball as a function of system parameters and how to maximize this speed. Adjustable parameters include the number of balls on the firing side of the cannon, the size of the magnet, the radius of the balls, and the incoming speed of the trigger ball. Earlier works have measured the force on the steel balls as a function of distance to compute the potential energies of the trigger ball and the ejected ball, thereby estimating the gained kinetic energy of the system $[1,2]$. In this report we present a more detailed analysis of the firing process and a novel approach to determine coefficients of restitution. The

\footnotetext{
* e-mail: akean@student. chalmers.se
}

structure is IMRaD plus model, dead end, and conclusion. Dead end contains discarded experiments and lines of thought that was not useful.

\section{Model}

The firing process is modeled as follows: the balls are subjected to magnetic force from the magnet, gravitational force from the earth and frictional and normal force from the track. Figure 1 presents the four different steps we consider in our model. (a) The trigger ball is attracted by the magnet, and rolls towards the bottom of the potential well with an initial velocity $v_{0}$. Assuming rolling without slipping, the sum of the translational and rotational energy is $\frac{7}{10} m v_{0}^{2}$ and the magnetic potential energy is 0 . (b) As the target ball hits the magnet, its magnetic potential energy is $E_{0}$ and its velocity is $v_{\text {in }}$. If the ball is still rolling without slipping, its rotational energy is $\frac{1}{5} m v_{\text {in }}^{2}$. However, the ball can also start sliding during its approach, in which case the rotational energy would be small than this value. Assuming that the rotation can not decrease as compared to the initial motion, a lower bound of the rotational energy is given by $\frac{1}{5} m v_{0}^{2}$. (c) As the kinetic energy of the target ball is transferred to the firing ball by collisions, the final ball of the line, with magnetic potential energy $E_{n}$, is ejected. Its velocity $v_{\text {out }}$ is expressed as a function of the total restitution coefficient $v_{\text {out }}=\varepsilon_{\text {tot }} v_{\text {in }}$. As no torque is applied to it, the eject ball has initially no rotation speed. (d) The ejected ball leaves the area of influence of the magnet, and is forced to roll by friction forces on the rail. We will assume that the energy lost in the process is negligible. 


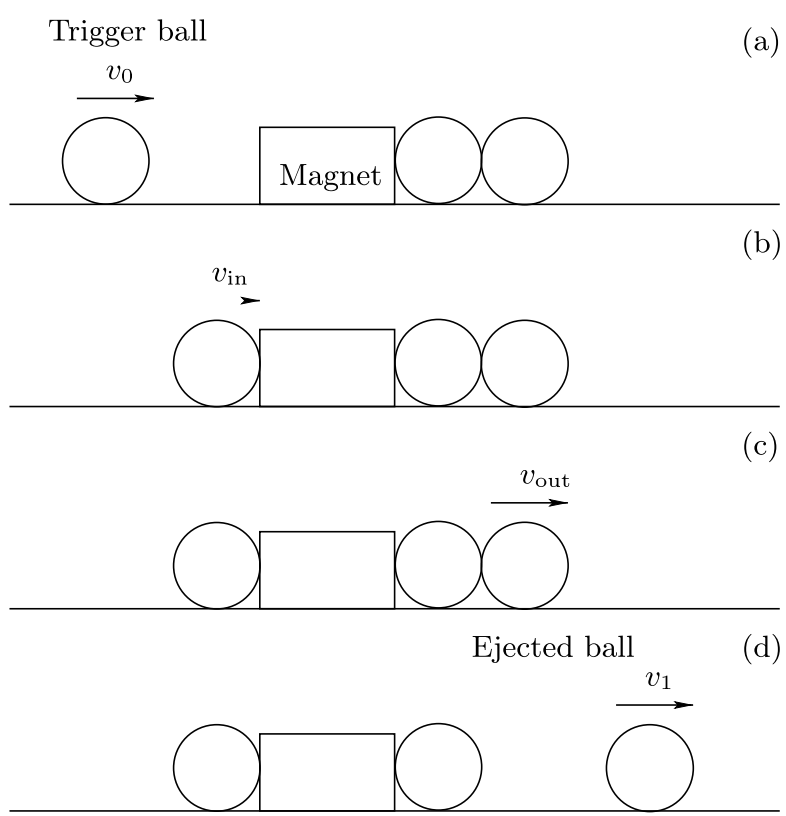

Fig. 1. The firing process of a Gaussian cannon. (a) A trigger ball moves towards a magnet and a line of steel balls. (b) The trigger ball collides with the magnet at speed $v_{\text {in }}$. The collision energy is transferred through the magnet and the balls in line. (c) The rightmost ball is ejected with an initial speed $v_{\text {out }}$. (d) The ejected ball reaches a speed of $v_{1}$.

In summary, we obtain the following system of equations from conservation of energy and the definition of the coefficient of restitution:

$$
\begin{gathered}
\frac{7}{10} m v_{0}^{2}=\alpha m v_{\text {in }}^{2}+E_{0}, \\
v_{\text {in }}^{2} \varepsilon_{\text {tot }}^{2}=v_{\text {out }}^{2}, \\
\frac{1}{2} m v_{\text {out }}^{2}+E_{n}=\frac{7}{10} m v_{1}^{2},
\end{gathered}
$$

with $\alpha=\frac{1}{2}+\frac{1}{5}\left(\frac{r \omega_{\text {in }}}{v_{\text {in }}}\right)^{2}$ describing the unknown rotational energy of the trigger ball. $\alpha=\frac{7}{10}$ corresponds to a no-slip rotation, i.e. $v_{\text {in }}=r \omega_{\text {in }}$. The lowest possible value for $\alpha$ is $\frac{1}{2}+\frac{1}{5}\left(\frac{v_{0}}{v_{\text {in }}}\right)^{2}$ (no angular acceleration). From equation (1) and $\omega_{0}<\omega_{\text {in }}$, we can prove

$$
m v_{\text {in }}^{2}+2 E_{0}<m v_{0}^{2}<m v_{\text {in }}^{2} .
$$

Therefore, if $m v_{0}^{2} \gg E_{0}$ then $v_{0} \approx v_{\text {in }}$ and $\alpha \approx \frac{7}{10}$. This will also be proven experimentally. Until then, the composite formula for $v_{1}\left(v_{0}\right)$ is: equations $(1),(2)$, and (3) together give:

$$
v_{1}^{2}=\left(\frac{\varepsilon_{\mathrm{tot}}^{2}}{2 \alpha}\right) v_{0}^{2}+\left(\frac{10 E_{n}}{7 m}-\frac{5 \varepsilon_{\mathrm{tot}}^{2} E_{0}}{7 \alpha m}\right) .
$$

This relation suggests a linear relation between the squared velocities, and depends on three parameters describing the kinetic energy of the trigger ball $\alpha$, the energy loss occurring during the energy transfer $\varepsilon$ and the

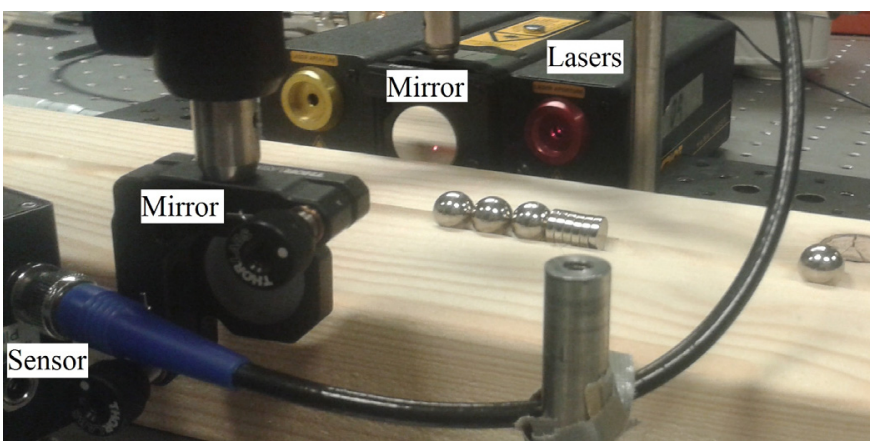

Fig. 2. An experimental setup used to measure speed. The Gaussian cannon is resting on a wooden track. A trigger ball is approaching the cannon from the right and on the left lasers are arranged in order to measure the firing velocity.

potential energy experienced by the balls $E_{n}$. In the following, we will estimate experimentally these terms to gain understanding on the problem, and verify the linear relationship between $v_{1}^{2}$ and $v_{0}^{2}$.

\section{Methods}

\subsection{Velocity measurement}

The experimental setup for velocity measurement consisted of a Gaussian cannon on a wooden track (of species fir). In order to measure speed, lasers were directed across the track and an oscilloscope registered whenever a ball interrupted a laser beam, and output time between interruptions. As the speed was expected to decrease due to friction, several lasers were used. Figure 2 presents an annotated picture of the setup used. Two laser beams crossed the path at five different points in total, the closest being $10 \mathrm{~mm}$ from the to-be ejected ball, i.e. at $x=10 \mathrm{~mm}$. The speed was obtained from $v=\left(x-x^{\prime}\right) /\left(t-t^{\prime}\right)$ where $x-x^{\prime}$ denotes the distance between two nearby beams and $t$ and $t^{\prime}$ denotes the corresponding times as the beams were disrupted by a fired ball. As $x-x^{\prime}$ is constant and $t-t^{\prime}$ is measured with nanosecond precision, the measurement error is dominated by the random nature of the firing. How early the ball touches the track appears to matter, and we could not fully control this. To combat this, all experiments with controllable parameters were repeated five times. For such measurements, the standard error of mean is displayed as error bars.

The magnet was made of several connected cylindrical neodymium magnets of radius $10 \mathrm{~mm}$ and height $2.5 \mathrm{~mm}$, magnetized along the symmetry axis [3]. Both big and small balls were used, with ball diameter $12.0 \mathrm{~mm}$ and $9.5 \mathrm{~mm}$ (errors being less than $1 \mu \mathrm{m}$ ) and mass $7.10 \mathrm{~g}$ and $3.51 \mathrm{~g}$ respectively (errors being less than $0.01 \mathrm{~g}$ ). The wooden track was U-shaped with slightly larger radius than the balls. Hot melt adhesive was used to fasten the magnet.

\subsection{Force measurement and energy determination}

To determine the energy $-E_{n}$ required to fully dislodge a ball from the system, consisting of a magnet and $n$ other balls stuck to it, the attractive force $F(d)$ was measured for 


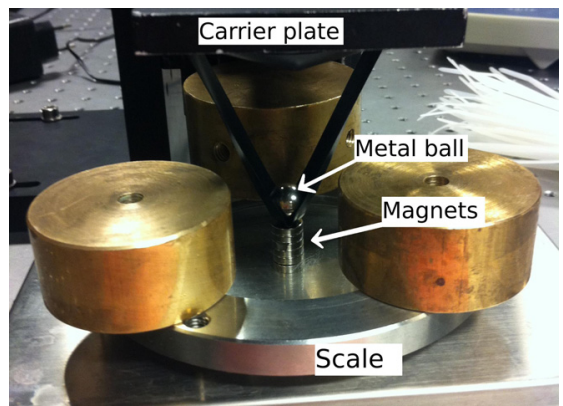

Fig. 3. The measurement setup for the force applied by magnets on a single steel ball. The ball is suspended above the magnets, which are attached to weights. The reciprocal force working on the magnets reduce the weight displayed on the scale, allowing for calculation of the magnetic force as a function of the difference in displayed weight. Themethod by which the ball was suspended in this image is the one we used for very small distances $(d<3 \mathrm{~mm})$ between theball and the magnets.

varying distances $d . E_{n}$ could then be calculated by integrating $F_{n}(d)$ from $2 r n$ to $\infty$. The actual measurement was done in two slightly different ways.

For large distances, $d>3 \mathrm{~mm}$, the ball was stuck to a nonmagnetic horizontal plate, whose height $y$ could be adjusted with precision $\Delta y<0.01 \mathrm{~mm}$. Beneath the plate was the magnet and the other balls, anchored to a weight, making the total weight $M$. This system rested upon a scale, showing a value $m$ with precision $\Delta m<0.01 \mathrm{~g}$. The attractive force $F$ was calculated as $F=(M-m) g$. This method has good precision for both $d$ and $F$, but for $d<3 \mathrm{~mm} F$ becomes so great that the plate bends, and therefore the precision of $y$ and $d$ becomes too low.

For small distances, $d<3 \mathrm{~mm}$, tape strips with a total thickness of $d$ were pasted onto the cannon and the ball was initially resting on top of the stacked strips. The cannon was pressed against the scale using heavy weights. The ball was then pulled by elevating the same plate as above as shown in Figure 3. As the plate was elevated, the displayed mass $m$ gradually lowered, until it hit a certain $m_{c}$, at which the ball snapped loose, causing $m$ to rapidly increase. $F$ was calculated as the maximum decrease in weight, $F=\left(M-m_{c}\right) g$. This method has great precision for $d$, but a larger error for $F, \Delta F<0.1 \mathrm{~N}$.

\subsection{COR determination}

The $\operatorname{COR} \varepsilon_{\text {tot }}$ is defined as

$$
\varepsilon_{\text {tot }}=\frac{v_{\text {out }}}{v_{\text {in }}} .
$$

These velocities are hard to measure, since the magnetic field accelerates the balls rapidly over a short distance very close to the magnet or close to the line of balls. Therefore we measured $v_{0}$ and $v_{1}$ and used equations (1) and (3) to obtain $v_{\text {in }}$ and $v_{\text {out }}$. This was done for number of balls $n=1, \ldots, 7$. The number of cylinder magnets was also varied, in the interval 3-9.

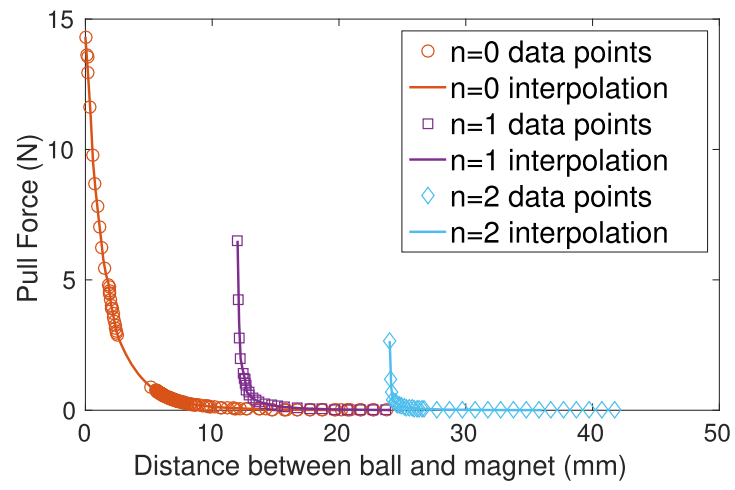

Fig. 4. Pull forces $F_{0}, F_{1}$, and $F_{2}$ for bigballs, as a function of distance $d$ between themagnet and the border of the ball. Note that $d$ is definedfrom the magnet itself, which is why $F_{n}$ is defined only for $d \geq n \cdot 12 \mathrm{~mm}$. $E_{n}$ was obtained by integrating the cubic interpolant (solid line).

\section{Results}

\subsection{Force and energy}

We generally define $E_{n}$ as the magnetic potential energy between the magnet and a firing ball having $n$ balls between itself and the magnet. For Figure 1, $n=1$. Note that we define the magnetic potential energy terms $E_{0}, E_{1}, \ldots$ so that they are always negative.

The force $F_{n}(d)$ was measured for small and big balls. For brevity, only the plot for big balls (Fig. 4) is shown. The plots were qualitatively similar. The energies $E_{n}$ in Table 1 was determined by integrating the cubic interpolant of $F_{n}(d)$. Higher $n$ results in less energy being needed to escape the cannon, which increases $v_{1}$.

\subsection{Velocity}

When measuring the final velocity $v_{1}$, the following parameters were varied:

- number of magnets;

- number of balls;

- ball size;

- trigger ball velocity $v_{0}$.

Figure 5 shows the two first parameters being varied. The 13 magnets data set shows that ejection velocity does not increase monotonously with the number of magnets. The optimal number of magnets was found to be 6 , adding more causes too much energy dissipation to be worth the extra magnetic flux. All following cannons will therefore consist of 6 magnets, in an effort to maximize velocities.

The influence of ball size can be seen in Figure 6. A smaller ball size allows higher $v_{1}$, and increase the optimal $n$. Small balls were used for most following experiments.

Finally, the parameter $v_{0}$ was varied, for virtually every data point in Figure 6. The result was qualitatively similar for every case, and therefore only one such graph is presented (Fig. 7). 
Table 1. Values of $E_{n}$ in mJ for big and small balls.

\begin{tabular}{lll}
\hline & Small balls & Big balls \\
\hline$E_{0}$ & -22 & -25 \\
$E_{1}$ & -2.9 & -4.6 \\
$E_{2}$ & -0.8 & -1.2 \\
$E_{3}$ & -0.2 & - \\
$E_{4}$ & -0.04 & - \\
\hline
\end{tabular}

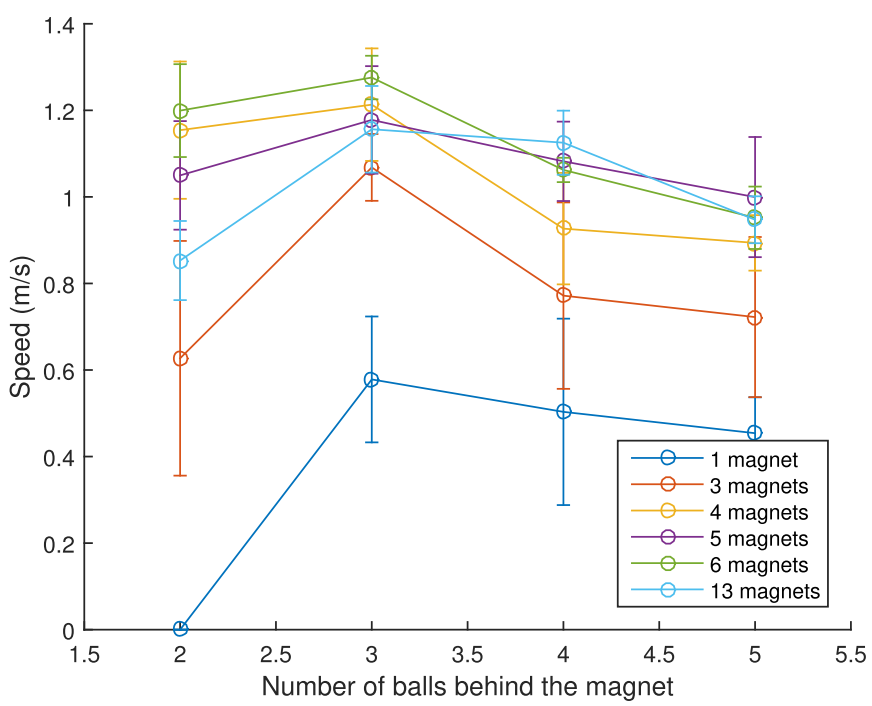

Fig. 5. Ejection velocities $v_{1}$ for small balls with a $v_{0}$ as small as possible, as a function of the number of balls and magnets.

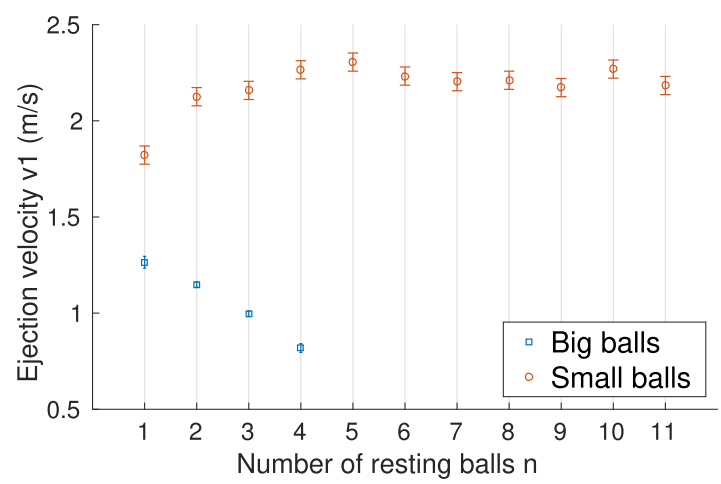

Fig. 6. Ejection velocities $v_{1}$ for balls of various sizes when $v_{0}=0$, as a function of the number of balls in-between the magnet and the ejected ball $n . n=0$ is left out, as no ejection occurs. For small balls, $n=5$ is optimal. For big balls, its $n=1$, the smallest possible $n$.

\subsection{COR}

$\log \left(\varepsilon_{\text {tot }}\right)$ as a function of $n$ is shown in Figure 8, with six magnets. The exponential relation suggests that $\varepsilon_{\mathrm{tot}}=\varepsilon_{\mathrm{bm}}^{2} \varepsilon_{\mathrm{bb}}^{n}$, where $\varepsilon_{\mathrm{bm}}$ is the COR between ball and magnet, and $\varepsilon_{\mathrm{bb}}$ is the COR between balls. Table 2 shows $\varepsilon_{\mathrm{bb}}$ and $\varepsilon_{\mathrm{bm}}$ for different number of magnets. The value of $\alpha$ affects how $v_{\text {in }}$ is calculated from $v_{0}$, and in turn $\varepsilon_{\text {tot }}$. Higher $n$ results in more energy being dissipated, which lowers $v_{1}$.

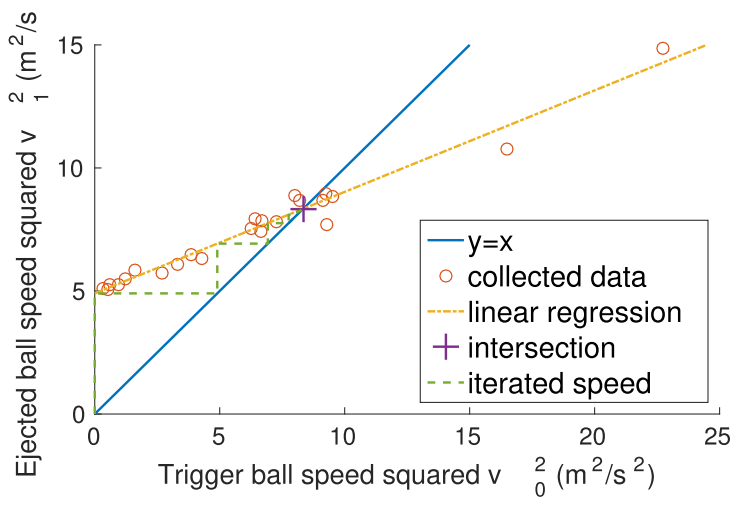

Fig. 7. Ejection velocity squared $v_{1}^{2}$ as a function of trigger velocity $v_{0}^{2}$, for small balls and $n=5$. Each circle corresponds to one ejected ball. The linear relation is a general feature, regardless of choice of ball size and $n$. Chained cannon: When the cannons are coupled together, the speed squared follows a convergent series, represented by the green dashed line. With a large number of cannons, the final speed corresponds to the purple intersection cross.

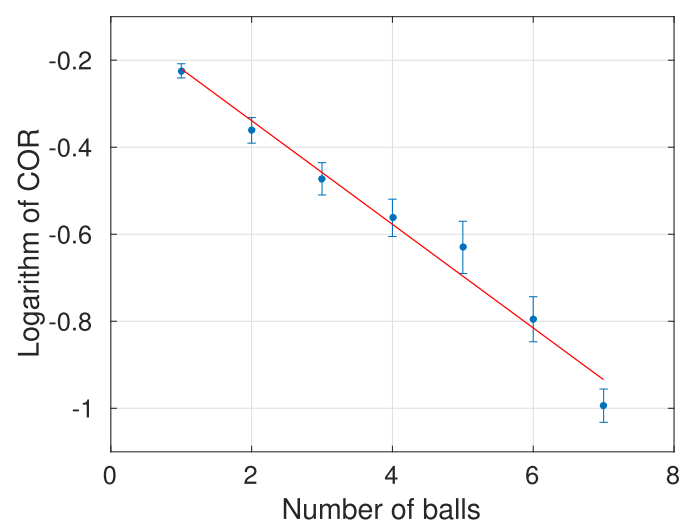

Fig. 8. The logarithm of COR values are shown on the $y$-axis and the number of balls are shown on the $x$-axis. The ball diameter was $12 \mathrm{~mm}$, the number of magnets was 6 and $\alpha=0.7$. The linear regression, $y=-0.12 n-0.08$, is shown as a solid red line.

\section{Discussion}

\section{$5.1 n$ dependence}

The general qualitative dependence on $n$ was as predicted by theory. For small $n$, the ejected ball transfers a significant amount of energy while escaping the magnet. For large $n$, a significant amount of energy is dissipated during the collisions between balls.

\subsection{Linearity}

Another strength of the model was the predicted (assuming constant $\alpha)$ and measured linearity between $v_{0}^{2}$ and $v_{1}^{2}$. Equations (1), (2), and (3) together gives a linear relation between $v_{1}$ and $v_{0}$, explicitly stated in equation (4). The linear relation between the squared velocities was tested for big and small balls with $n=3,4, \ldots, 7$. Figure 7 shows the test for small balls with $n=5$. The linear relation hold 
Table 2. COR values obtained from linearization of Figure 8 , under the assumption $\varepsilon_{\mathrm{tot}}=\varepsilon_{\mathrm{bm}}^{2} \varepsilon_{\mathrm{bb}}^{n}$.

\begin{tabular}{lllll}
\hline Number of magnets & $\varepsilon_{\mathrm{bb}}$ & $\begin{array}{l}\varepsilon_{\mathrm{bm}} \\
\alpha=0.5\end{array}$ & $\begin{array}{l}\varepsilon_{\mathrm{bm}} \\
\alpha=0.7\end{array}$ & Norm of residual \\
\hline 3 & 0.90 & 0.76 & 0.90 & 0.095 \\
4 & 0.88 & 0.89 & 1.06 & 0.12 \\
6 & 0.91 & 0.78 & 0.92 & 0.049 \\
6 & 0.89 & 0.78 & 0.93 & 0.096 \\
9 & 0.92 & 0.76 & 0.90 & 0.066 \\
\hline
\end{tabular}

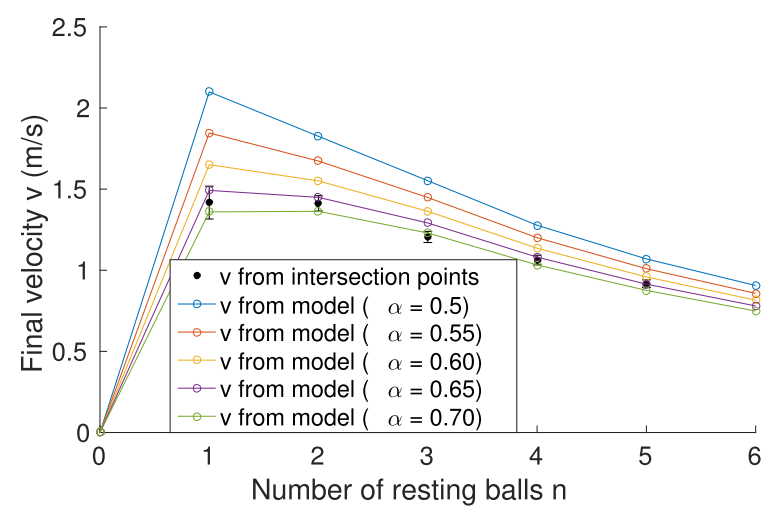

Fig. 9. Comparison between intersection points from linear regressions (yellow line in Fig. 7), and intersection points from our model (fix points from Eq. (4)). Since equation (4) depends on $\alpha$, which is not yet known, several plots are made depending on the choice of $\alpha$.

\subsection{Chained cannon}

When two cannons are coupled together such that $v_{1}$ of the first one becomes $v_{0}$ of the second, the result is a better cannon. With an arbitrarily long chain of cannons, what is the final velocity?

It is known that for a single cannon, $v_{n+1}^{2}$ is a linear function of $v_{n}^{2}$, in particular equation (4). In general, the sequence $x_{n+1}=A x_{n}+B$ converges to $\frac{B}{1-A}$ if $|A|<1$. For our system, $A=\frac{\varepsilon_{\text {tot. }}^{2}}{2 \alpha}$. This is positive and smaller than 1 , because $\varepsilon_{\text {tot }}<1$ and $2 \alpha>1$. Figure 7 shows the convergence of the series $v_{0}^{2}, v_{1}^{2}, \ldots$ To test this quantitatively, we compare the velocities from intersection points between linear regressions and $v_{0}=v_{1}$ (seen as the purple dot in Fig. 7) to calculated values, $v^{2}=\frac{B}{1-A}$. The comparison can be seen in Figure 9. It not only gives us the expected value $\alpha=0.7$, but can also be seen as a test of equation (4), if $\alpha=0.7$ is assumed.

Figure 9 suggests that $\alpha=0.7$ is a good approximation, which would allow equations (1) and (4) to be simplified. Analysis of the optimal $\alpha$ values show that they have mean 0.68 and estimated SD 0.028. Thus 0.7 is $0.74 \mathrm{SD}$ from the $\alpha$ distribution, which corresponds to failing to discard null hypothesis $\alpha=0.7$ with $p<0.5$. In summary, we can accept the approximation $\alpha=0.7$. An interesting observation of Figure 9 is that the optimal $n$ is lower than if a single cannon were used. This is due to the increased importance of energy dissipation in a chained system.

\section{Dead end}

Early on, we considered comparing measured pull force to calculated force from $B$-field equations. This approach was discarded, due to the increased complexity and the magnetic dipole moment being unknown.

We tried filming the ejected ball to learn about its rotation, but the time resolution was not good enough for quantitative analysis. Sometimes balls rotated around the vertical axis due to contact with the side of the firing track, which made it impossible to determine angular velocity.

\section{Conclusion}

The Gaussian canon is a simple device that constitutes an insightful optimization problem. The ejection of the firing ball is due to the large kinetic energy transferred by collisions from the trigger ball, and to the limitation of the magnetic potential energy by the spacing balls. While a large number of spacing balls decreases the depth of the potential well from which the firing ball has to escape, it also increases the energy loss occurring during the energy transfer. This two competing behaviors result in the existence of an optimal number of spacing balls.

We presented a simple model based on energy conservation to relate the velocity of the firing ball to that of the trigger ball, and found a linear relation between this two quantities squared. Our model relies on three parameters, namely a rotational kinetic term $\alpha$, the coefficient of restitution $\varepsilon_{\text {tot }}$ and the magnetic potential energy $E_{n}$, of which the latter two were investigated experimentally. For $\alpha$, we tested all possible values, and found in each case that with $\alpha=0.7$, the model agreed with experiments. That $\alpha=0.7$ also brought better understanding to the physics of the ejection process. The linear behavior was verified by the experiments, and shed light on the terminal velocity reached by a succession of Gaussian canon. This constitutes the main result of this work.

In addition to the optimization study presented here, the Gaussian canon exhibits numerous features that deserve attention. For instance, the symmetry between the number of trigger and firing ball (one and one in this work) can be seen as a generalization of Newton's cradle, but also studied as the propagation of a soliton wave through the spacing balls [4]. The inelastic collisions leading to finite losses in the coefficient of restitution also constitutes a problem of interest. We therefore believe that the Gaussian canon is a powerful tool not only to fire metallic balls, but also to teach and understand physics. 


\section{References}

1. J.A. Rabchuk, The gauss rifle and magnetic energy, Phys. Teach. 41, 158 (2003)

2. D. Kagan, Energy and momentum in the gauss accelerator, Phys. Teach. 42, 24 (2004)
3. Neodymmagnet, product number 31-1467, http://www.clasohlson.com

4. The sine-Gordon model and its applications (2014), http://www.springer.com/in/book/9783319067216

Cite this article as: Åke Andersson, Carl-Joar Karlsson, Hampus Lane, The Gaussian cannon, Emergent Scientist 1, 6 (2017) 